\title{
Meniscal "Ramp" Lesions: Surgical Incidence and the Development of Magnetic Resonance Imaging Diagnostic Criteria
}

\author{
Matthew Greenaway, M.B.B.S.(Hons), Edward Walton, M.B.Ch.B., F.R.C.R., \\ Daren Gibson, M.R.C.S., B.Sc., F.R.C.R., F.R.A.N.C.R., \\ Anton Le Roux, B.V.Sc., M.R.C.V.S., M.B.B.Ch., M.M.E.D.Rad.D., F.R.A.N.Z.C.R., \\ Piers Yates, M.B.B.S.(Hons), B.Sc.(Hons), M.R.C.S.(Eng), F.R.C.S.(TrGOrth), \\ F.R.A.C.S.(Ortho), F.A.Orth.A., Jay Ebert, Ph.D., and \\ Peter D'Alessandro, M.B.B.S. Hons.(UWA), F.R.A.C.S., F.A.Orth.A.
}

Purpose: To establish the magnetic resonance imaging (MRI) and operative incidence of ramp lesions in a consecutive group of patients who had undergone anterior cruciate ligament reconstruction (ACLR) and to propose a graded radiologic classification that seeks to define key preoperative features that most strongly correlate with arthroscopically confirmed ramp lesions. Methods: After retrospective review, all consecutive patients who underwent ACLR with preoperative MRI over a 16-month period were included in the study. Diagnostic arthroscopy with direct visualization of the posteromedial compartment was used to establish the true incidence of ramp lesions. Three radiologists, blinded to the surgical findings, independently analyzed the MRI scans of all patients and classified the signal intensity at the posterior meniscocapsular junction using 4 criteria (type 1 , diffuse increased signal equal to adjacent femoral cartilage; type 2, diffuse increased signal greater than cartilage; type 3, diffuse increased signal plus full-height linear fluid-signal cleft; and type 4, full-height fluid-signal meniscocapsular separation). Interobserver reliability, in addition to sensitivity, specificity, and positive and negative predictive values, was calculated. Results: Of the 93 patients included (mean age, 26.7 years), 11 (11.8\%) were confirmed to have ramp lesions on arthroscopy. Inter-rater reliability for the MRI diagnosis of the positive ramp lesions was good, at 0.75 . Type 3 criteria were the most accurate, producing average sensitivity and specificity values of $85 \%$ and $82 \%$, respectively. Conclusions: Ramp lesions are not infrequently encountered in ACLR and, if not recognized at the time of surgery, may contribute to inferior outcomes. MRI is an effective method of preoperative assessment, and the presence of type 3 changes should be used as criteria for radiologically definitive ramp lesion diagnosis. Clinical Relevance: This study highlights the incidence of meniscal ramp lesions encountered in ACLR surgery and the effectiveness of MRI as a preoperative imaging modality.

From Fiona Stanley Fremantle Hospitals Group, Perth, Australia (M.G., E.W., D.G., A.L., P.Y., P.D.); Orthopaedic Research Foundation of Western Australia, Perth, Australia (M.G., P.Y., J.E., P.D.); North Bristol NHS Trust, Bristol, England (E.W.); School of Surgery, University of Western Australia, Perth, Australia (P.Y., P.D.); and School of Human Sciences (Exercise and Sport Science), University of Western Australia, Perth, Australia (J.E.).

The authors report no conflicts of interest in the authorship and publication of this article. Full ICMJE author disclosure forms are available for this article online, as supplementary material.

Received August 16, 2019; accepted March 12, 2020.

Address correspondence to Matthew Greenaway, M.B.B.S.(Hons), 11 Robin Warren Dr, Murdoch,WA 6150,Australia.E-mail: matthewjgreenaway@ gmail.com

Crown Copyright $(\subset) 2020$ Published by Elsevier on behalf of the Arthroscopy Association of North America. This is an open access article under the CC BYNC-ND license (http://creativecommons.org/licenses/by-nc-nd/4.0/).

2666-061X/19865

https://doi.org/10.1016/j.asmr.2020.03.003
W hereas there is an increasing appreciation of the link between an array of intra-articular knee pathologies and abnormal knee biomechanics, the strong association between anterior cruciate ligament (ACL) rupture and meniscal injury is well known. ${ }^{1,2}$ One under-recognized meniscal lesion involves disruption of the posterior meniscocapsular junction of the medial meniscus, or a "ramp lesion." setting of ACL reconstruction (ACLR), biomechanical cadaveric studies have suggested that untreated ramp lesions may result in increased anterior tibiofemoral joint laxity, preventing restoration of normal knee joint kinematics., 6

Ramp lesions can be easily overlooked during standard diagnostic knee arthroscopy because of a potential blind spot when viewing the medial compartment; 


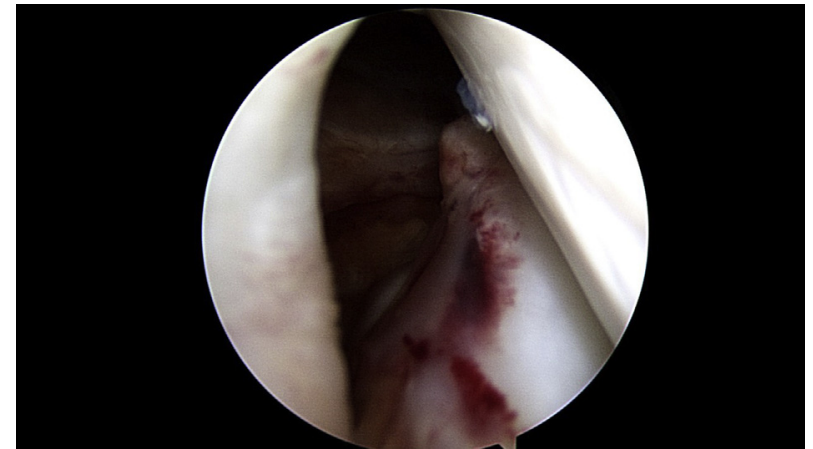

Fig 1. Arthroscopic image showing a ramp lesion as visualized via the intercondylar notch view.

therefore, formal arthroscopy in the posteromedial compartment has been recommended for surgical diagnosis. ${ }^{2,7}$ Formal investigation in the posteromedial compartment during ACLR may not be routine surgical practice, reinforcing the importance of accurate preoperative detection of these lesions on magnetic resonance imaging (MRI). When compared with the gold standard of arthroscopy, the effectiveness of MRI for the detection of ramp lesions has only recently been explored. Existing literature has generally focused on repair techniques and surgical outcomes, with a relative paucity of literature evaluating the accuracy of MRI for ramp lesion diagnosis. ${ }^{8,9}$

The purposes of this study were to establish the MRI and operative incidence of ramp lesions in a consecutive group of patients who had undergone ACLR and to propose a graded radiologic classification that seeks to define key preoperative features that most strongly correlate with arthroscopically confirmed ramp lesions. The hypothesis was that ramp lesions would frequently be encountered during ACLR and that MRI would be an effective imaging modality when diagnostic criteria were used.

\section{Methods}

This study was approved by the relevant human research ethics committee. Over a 16-month period (September 2016 to January 2018), all consecutive patients who underwent ACLR (as part of primary, revision, or multiligament surgery) and underwent preoperative MRI of the affected knee were included in the study. Patients undergoing revision were excluded if they underwent a previous ramp lesion repair.

\section{Surgical Technique}

All surgical procedures were performed by a single surgeon (P.D.) with fellowship training in sports knee surgery. To grade the laxity of the cruciate and/or collateral ligaments, all knees were examined with patients under anesthesia. Patients were placed supine on the operating table, with a foot bolster at $90^{\circ}$ of flexion and side support allowing full range of motion. Diagnostic arthroscopy from an anterolateral portal was performed, including standard assessments of the medial, lateral, and patellofemoral compartments. The leg was then allowed to hang over the side of the operating table at approximately $40^{\circ}$ of flexion, and a mild valgus force was applied. The triangular space between the posterior cruciate ligament, tibial plateau, and medial femoral condyle was visualized, and the arthroscope was gently introduced via the intercondylar notch, through this triangle, into the posteromedial compartment of the knee. The presence of a ramp lesion was determined if there was a longitudinal separation "valley" between the peripheral attachment of the medial meniscus and the capsule (Fig 1); this was formally assessed with either an arthroscopic probe or an 18gauge spinal needle. If required for safe access, a controlled release of the deep medial collateral ligament was performed using an 18-gauge needle to perform "pie crusting" of the ligament from outside in, until adequate visualization was achieved. If a ramp lesion was discovered, it was repaired in all cases (Fig 2).

\section{MRI Evaluation}

Routine preoperative MRI studies of the included patients were acquired from 3 radiology departments. These images were subsequently uploaded to a single viewing platform for analysis. In all patients, MRI examinations were performed using a 1.5-T scanner with a dedicated knee coil and the knee imaged at nearly complete extension. Obligatory sequences included contiguous sagittal $\mathrm{T} 2$ fat-saturated proton density (PD) images.

Three fellowship-trained subspecialty musculoskeletal radiologists (E.W., D.G., A.L.) with 22, 15, and 6 years of radiology experience, who were blinded to the intraoperative findings, independently reviewed all the MRI scans of each patient. Specifically, MRI scans were assessed according to the presence and morphology of fluid signal at the posterior meniscocapsular junction of the medial meniscus on sagittal $\mathrm{T} 2$ fat-saturated PD

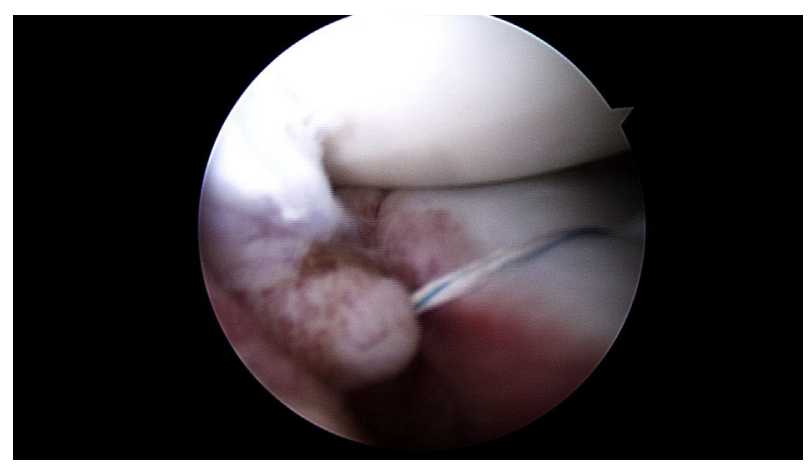

Fig 2. Arthroscopic image showing a repaired ramp lesion. 


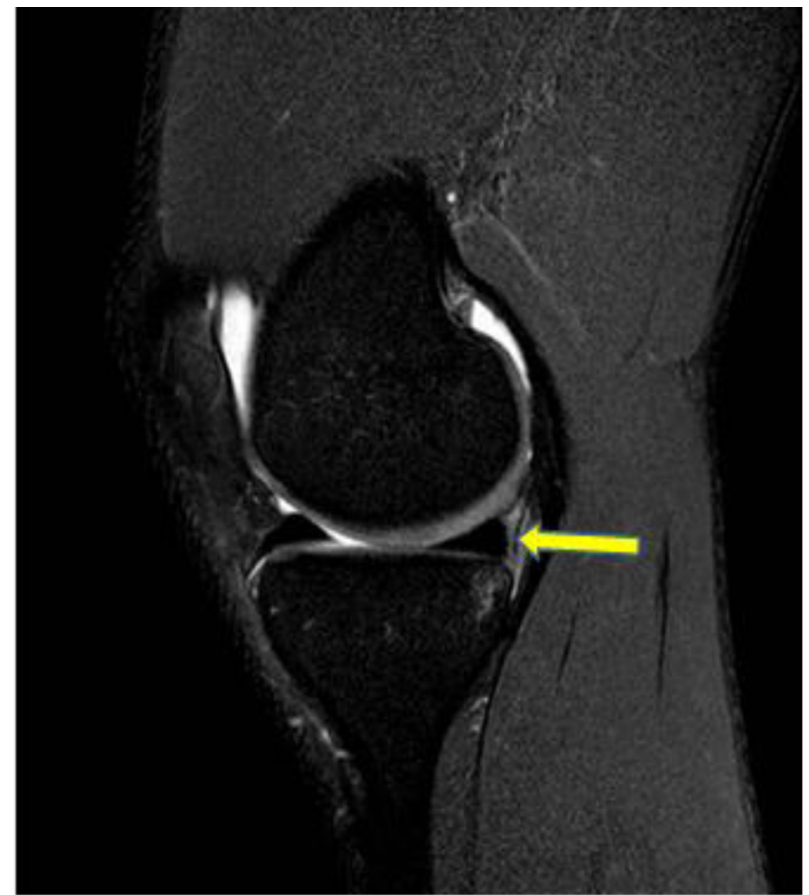

Fig 3. Type 1 grading, indicative of diffuse increased signal (arrow) equal to the adjacent femoral cartilage.

images. On the basis of experience, we developed a classification system for the vertically orientated signal characteristics at the posterior medial meniscocapsular junction, with grades ranging from type 1 to type 4 . Whereas signal intensity less than the adjacent cartilage was classified as normal, the grades were classified as follows: type 1, diffuse increased signal equal to the adjacent femoral cartilage (Fig 3); type 2, diffuse increased signal greater than the adjacent cartilage (Fig 4); type 3, diffuse increased signal plus full-height linear fluid-signal cleft (Fig 5); and type 4, full-height fluidsignal meniscocapsular separation (Fig 6). "Full height," indicated in types 3 and 4, describes complete involvement of the junction with no intact tissue identified. The presence of coexisting injuries to the posterior oblique ligament (POL), true intrameniscal medial tears, and injury to the medial collateral ligament was simultaneously recorded.

\section{Statistical Analysis}

The incidence of meniscal ramp lesions on both preoperative MRI and intraoperative evaluation was determined. Intraclass correlation coefficient values were produced to assess interobserver reliability of the MRI assessment for the 3 independent readers. The sensitivity, specificity, negative predictive value (NPV), and positive predictive value (PPV) for the grading of ramp lesions were also calculated. Statistical analyses were performed using MedCalc for Windows (version 18.5; MedCalc Software, Ostend, Belgium). Statistical significance was determined at $P<.05$.

\section{Results}

\section{Study Population}

Over the 16-month study period, 93 patients underwent ACLR and were included in the study. The study population included 34 female and 59 male patients, and the average age at surgery was 26.7 years. The indications for surgery included primary ACLR $(\mathrm{n}=74)$, revision ACLR $(\mathrm{n}=8)$, and multiligament reconstruction $(\mathrm{n}=11)$.

\section{Surgical Incidence}

Of the 93 cases included in this analysis, 11 (11.8\%) had an arthroscopically confirmed ramp lesion. The mean time interval between preoperative MRI and the surgical procedure was 79 days (range, 20-177 days).

\section{MRI Ramp Lesion Classification}

The inter-rater reliability for all 93 cases was moderate, at 0.65 , with the inter-rater reliability for the 11 positive ramp lesions being good, at $0.75 .{ }^{10}$ Sensitivity, specificity, PPV, and NPV are shown in Table 1 for each of the 4 classification types. Type 3 criteria proved to be the most accurate, producing sensitivity values with an average of $85 \%$ (95\% confidence interval [CI], $58 \%-98 \%)$ and specificity of $82 \%$. Furthermore, an average PPV of $41 \%$ and NPV of up to

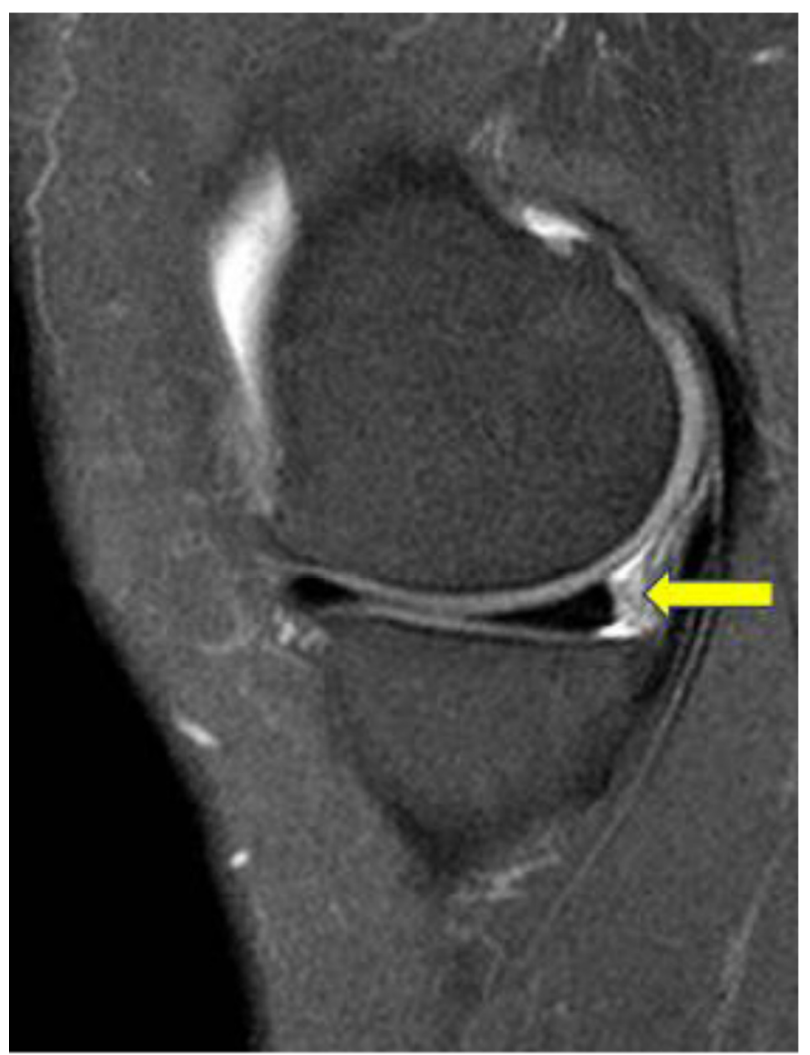

Fig 4. Type 2 grading, indicative of diffuse increased signal (arrow) greater than the adjacent cartilage. 


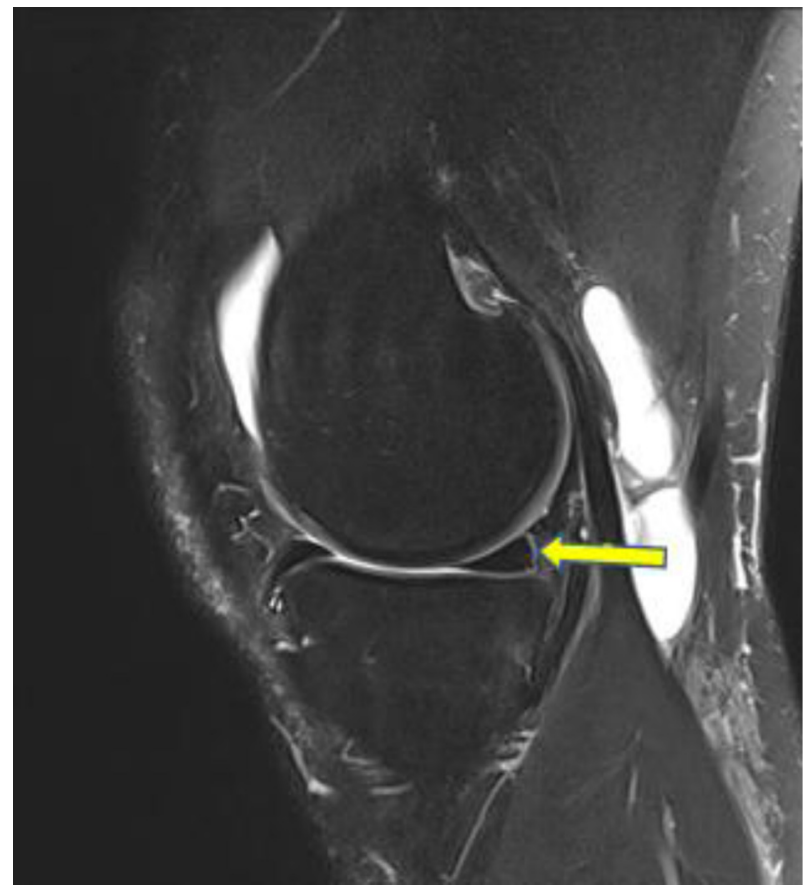

Fig 5. Type 3 grading, indicative of diffuse increased signal plus full-height linear fluid-signal cleft (arrow).

97\% (95\% CI, 92\%-99\%) for type 3 criteria were observed. Type 4 criteria with evidence of definitive meniscocapsular separation had a very high specificity of up to $90 \%$ but poor sensitivity averaging $58 \%$. Types 1 and 2 had excellent sensitivity, at 100\%, because all ramp lesions showed at least increased $\mathrm{T} 2$ signal at the meniscocapsular junction. The specificity, however, was poor, with average values of $17 \%$ and $47 \%$, respectively. The NPV was $100 \%$ for both types. POL injury had a moderate positive correlation with ramp lesions, with a Pearson correlation coefficient of 0.39 $(P=.0001 ; \quad 95 \% \quad C I, 0.24-0.54)$ for at least intermediate-grade POL injury. The 11 positive ramp lesions had a prevalence rate of concurrent intrameniscal tears of $27 \%$ compared with a rate of $46 \%$ in patients without ramp lesions. This, however, was of limited statistical significance, with a correlation coefficient of only -0.12 ( $P=.23 ; 95 \% \mathrm{CI},-0.39$ to 0.08$)$.

\section{Discussion}

In this study, $11.8 \%$ of patients had a confirmed ramp lesion on arthroscopy. Radiologically, inter-rater reliability for the detection of positive ramp lesions was good, at 0.75. Type 3 criteria, with diffuse increased signal plus full-height linear fluid-signal cleft, proved most accurate on MRI, with sensitivity of $85 \%$ and specificity of $82 \%$.

Although arthroscopy remains the gold standard for diagnosing a ramp lesion, standard anteromedialcompartment visualization is insufficient and the posterior meniscocapsular junction should be directly visualized using an intercondylar notch view. This, however, may not be routine clinical practice, reinforcing the importance of preoperative radiologic diagnosis.

The ramp lesion surgical incidence of $11.8 \%$ in our study is consistent with results reported in the literature. Previous studies investigating operatively confirmed ramp lesions report a surgical incidence between 9.3\% and $29.7 \% .^{2,3,7,11}$ In one study, Sonnery-Cottet et al. ${ }^{2}$ found that among 125 patients who had medial meniscal tears, only $60 \%$ of these tears were detected using standard anterior portals. An additional 23.2\% were diagnosed by direct visualization of the posteromedial compartment using an intercondylar notch view, and a further $16 \%$, using a posteromedial portal.

Our study aimed to determine an appropriately sensitive and specific imaging classification system to increase the confidence of preoperative ramp lesion diagnosis. The findings seen in type 3 and 4 lesions support the previously established criterion of a fluidsignal cleft as the most specific finding of an unstable meniscocapsular injury. ${ }^{12-14}$ Investigating the ability of MRI to detect ramp lesions in patients undergoing ACLR, Arner et al. ${ }^{8}$ reported a sensitivity of $53.9 \%$ to $84.6 \%$ with a specificity of $92.3 \%$ to $98.7 \%$. In a study undertaken by DePhillipo et al., ${ }^{9}$ it was concluded that MRI performed poorly (48\% sensitivity) in detecting these specific injuries and that a focused intraoperative assessment was essential. A more recent study by Hatayama et al. ${ }^{11}$ using a criterion of "high signal

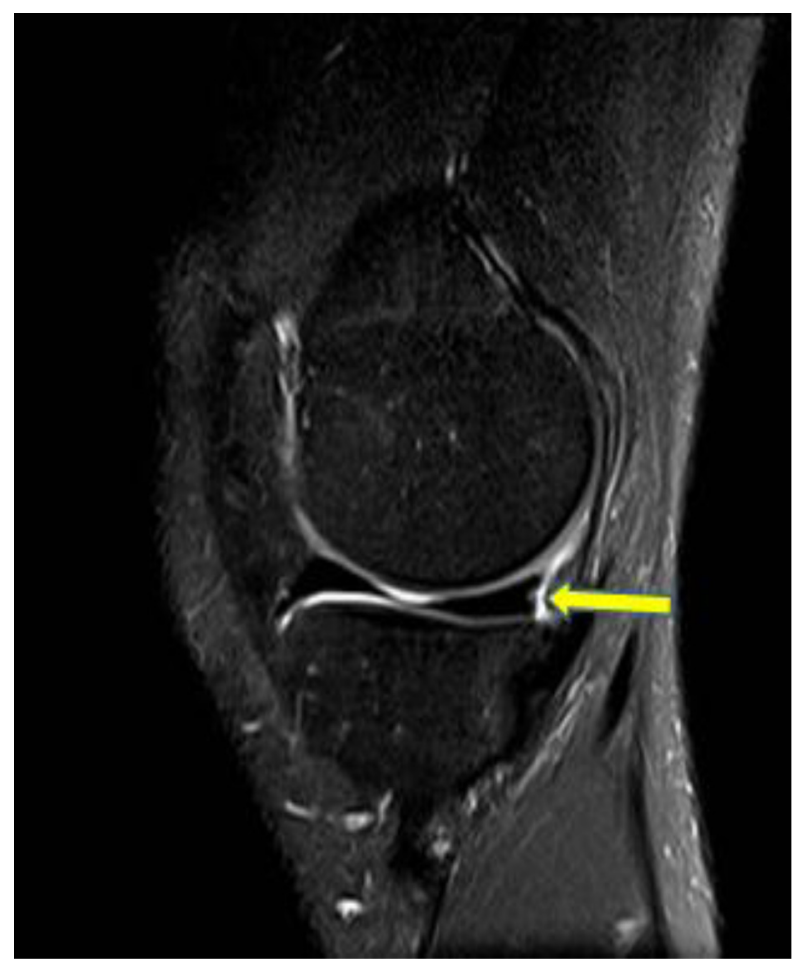

Fig 6. Type 4 grading, indicative of full-height fluid-signal meniscocapsular separation (arrow). 


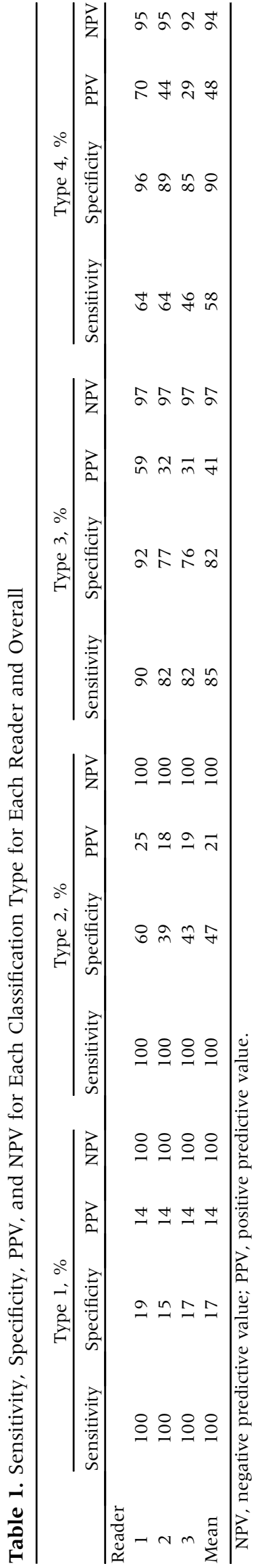

irregularity" to diagnose a ramp lesion reported sensitivity values of $83 \%$ and $67 \%$ using 3 - and 1.5 -T MRI scanners, respectively.

In our study, the presence of diffuse increased signal (type 2) but no definite linear fluid signal was encountered in most cases and yielded a very high sensitivity value of $100 \%$. This is pathologically likely to represent potentially reversible post-traumatic edema at the meniscocapsular junction. On the basis of the findings of this study, we believe that when reporting these MRI studies, it is important to highlight type 2 changes to forewarn the surgeon of a potential occult ramp lesion.

As supported throughout the literature and confirmed in our study, MRI has proved to be an effective tool to exclude ramp lesions. ${ }^{8}$ In the practice of the senior author (P.D.), altered fluid signal at the posterior meniscocapsular junction is often encountered in the presence of acute traumatic internal derangements, making it difficult to dichotomously determine what exactly constitutes "abnormal." This study shows that signal intensity less than or equal to the adjacent cartilage on T2 PD sequences can consistently exclude a ramp lesion with an NPV of $100 \%$. However, this criterion alone will considerably reduce overall sensitivity.

Given the limited knowledge regarding ramp lesions, the risk factors for development of a posteromedial meniscocapsular injury are not well established. One study has attributed medial meniscal slope to an increased propensity for development of a ramp lesion: Among 53 patients with an arthroscopically confirmed ramp lesion, preoperative MRI showed that medial meniscal slope was increased by at least $1.5^{\circ}$ compared with individuals with an isolated ACL injury. ${ }^{15}$

\section{Limitations}

The major limitation of this study is the inclusion of patients undergoing revision ACLR or multiligament reconstruction in the study group. Although no patients who underwent a previous ramp lesion repair were included, the aforementioned patients are surgically and radiologically more complex. Another limitation is the small sample size. In addition, the MRI examinations were performed on 2 different 1.5-T MRI scanners from different vendors, at different institutions, a potentially confounding variable. The mean time interval from MRI to arthroscopy was 79 days, and this potentiates the subsequent degenerative morphologic changes in meniscocapsular injuries prior to definitive arthroscopy.

\section{Conclusions}

Ramp lesions are not infrequently encountered in ACLR and, if not recognized at the time of surgery, may contribute to inferior outcomes. MRI is an effective method of preoperative assessment, and the presence of 
type 3 changes should be used as criteria for radiologically definitive ramp lesion diagnosis.

\section{References}

1. De Smet A, Graf B. Meniscal tears missed on MR imaging: Relationship to meniscal tear patterns and anterior cruciate ligament tears. AJR Am J Roentgenol 1994;162:905-91 1.

2. Sonnery-Cottet B, Conteduca J, Thaunat M, Gunepin FX, Seil R. Hidden lesions of the posterior horn of the medial meniscus: A systematic arthroscopic exploration of the concealed portion of the knee. Am J Sports Med 2014;42: 921-926.

3. Liu $X$, Feng $H$, Zhang $H$, Hong L, Wang XS, Zhang J. Arthroscopic prevalence of ramp lesion in 868 patients with anterior cruciate ligament injury. Am J Sports Med 2011;39:832-837.

4. Strobel M. Manual of arthroscopic surgery. New York: Springer, 1988.

5. Peltier A, Lording T, Maubisson L, Ballis R, Neyret P, Lustig S. The role of the meniscotibial ligament in posteromedial rotational knee stability. Knee Surg Sports Traumatol Arthrosc 2015;23:2967-2973.

6. Stephen JM, Halewood C, Kittl C, Bollen SR, Williams A, Amis AA. Posteromedial meniscocapsular lesions increase tibiofemoral joint laxity with anterior cruciate ligament deficiency, and their repair reduces laxity. Am J Sports Med 2016;44:400-408.

7. Bollen SR. Posteromedial meniscocapsular injury associated with rupture of the anterior cruciate ligament: A previously unrecognised association. J Bone Joint Surg Br 2010;92:222-223.

8. Arner JW, Herbst E, Burnham JM, et al. MRI can accurately detect meniscal ramp lesions of the knee. Knee Surg Sports Traumatol Arthrosc 2017;25:3955-3960.

9. DePhillipo NN, Cinque ME, Chahla J, Geeslin AG, Engebretsen L, LaPrade RF. Incidence and detection of meniscal ramp lesions on magnetic resonance imaging in patients with anterior cruciate ligament reconstruction. Am J Sports Med 2017;45:2233-2237.

10. Koo T, Mae YL. A guideline of selecting and reporting intraclass correlation coefficients for reliability research. J Chiropr Med 2016;15:155-163.

11. Hatayama K, Terauchi M, Saito K, Aoki J, Nonaka S, Higuchi H. Magnetic resonance imaging diagnosis of medial meniscal ramp lesions in patients with anterior cruciate ligament injuries. Arthroscopy 2018;34: $1631-1637$.

12. House CV, Connell DA, Saifuddin A. Posteromedial corner injuries of the knee. Clin Radiol 2007;62: 539-546.

13. Yeo Y, Ahn J, Kim H, et al. MR evaluation of the meniscal ramp lesion in patients with anterior cruciate ligament tear. Skeletal Radiol 2018;47:1683-1689.

14. Hash T. Magnetic resonance imaging of the knee. Sports Health 2013;5:78-107.

15. Song GY, Liu X, Zhang H, et al. Increased medial meniscal slope is associated with greater risk of ramp lesion in noncontact anterior cruciate ligament injury. Am J Sports Med 2016;44:2039-2046. 